\title{
Carotid artery stenting with contralateral carotid occlusion in a rare aortic arch configuration Umberto M. Bracale ${ }^{a}$, Felice Pecoraro ${ }^{a}$, Marco Caruso ${ }^{b}$, Gaetano Vitale ${ }^{a}$ and
Guido Bajardi $^{\mathrm{a}}$
}

We present the case of a 47-year-old man admitted to our department with an episode of aphasia. Duplex scan showed an occluded right internal carotid artery and severe left internal carotid artery stenosis. Contrastenhanced computer tomography demonstrated a common trunk for both common carotid arteries anterior to the trachea and aberrant right subclavian artery posterior to the esophagus. The patient was considered to be a high risk for carotid endarterectomy and, consequently, we performed stenting of the left carotid artery. To our knowledge, this is the first case reporting the combination of these two aortic arch anomalies and the concomitant endovascular treatment of atherosclerotic carotid stenosis.

\author{
J Cardiovasc Med 11:628-630 (c) 2010 Italian Federation of \\ Cardiology.
}

Journal of Cardiovascular Medicine 2010, 11:628-630

Keywords: aortic arch anomalies, carotid artery stenting, carotid occlusion, carotid stenosis

${ }^{a}$ Vascular and Endovascular Surgery Unit and ${ }^{b}$ Invasive Cardiology Unit, University of Palermo, Palermo, Italy

Correspondence to Umberto M. Bracale, Vascular and Endovascular Surgery Unit, University of Palermo, 90127 Palermo, Italy

Tel: +39 091655 2765; fax: +39 091655 2636; e-mail: umbracale@unipa.it

Received 3 October 2009 Revised 29 October 2009

Accepted 15 December 2009

stenosis under distal embolic protection with SpiderFx $6 \mathrm{~mm}$ (EV3, Minneapolis, Minnesota, USA). Postdilatation was achieved with $6 \times 20 \mathrm{~mm}$ Maverick balloon (Boston Scientific, Natick, Massachusetts, USA). The final carotid angiogram was satisfactory (Fig. 2b) and the control cerebral angiogram revealed no changes from the basal (Fig. 3). The patient was discharged the next day on dual antiplatelet therapy. A follow-up CTA 3 months after the procedure showed the stent to be in good position with no restenosis (Fig. 2c).

\section{Discussion}

Aberrant RSCA associated with common carotid trunk is rare and its incidence is reported to be between $0.05 \%$ and $0.16 \%[1,2]$. A few cases have been published describing this anomaly but no one has reported the treatment of concomitant ICA stenosis [1-4].

Left aortic arch with aberrant RSCA and common carotid trunk is exceptionally reported in the literature. It is supposed that selective regression of various parts of the embryologic double aortic arch could explain this anomaly [1]. Recently, Natsis et al. [2] reported one case $(0.16 \%)$ in their series of 633 consecutive arch angiographies but a real incidence is not known because it remains asymptomatic in most cases. However, when symptomatic, dysphagia due to posterior compression of the esophagus by the aberrant RSCA is the most common manifestation, whereas the common carotid trunk may rarely cause tracheal compression in elderly patients [3]. 
Fig. 1

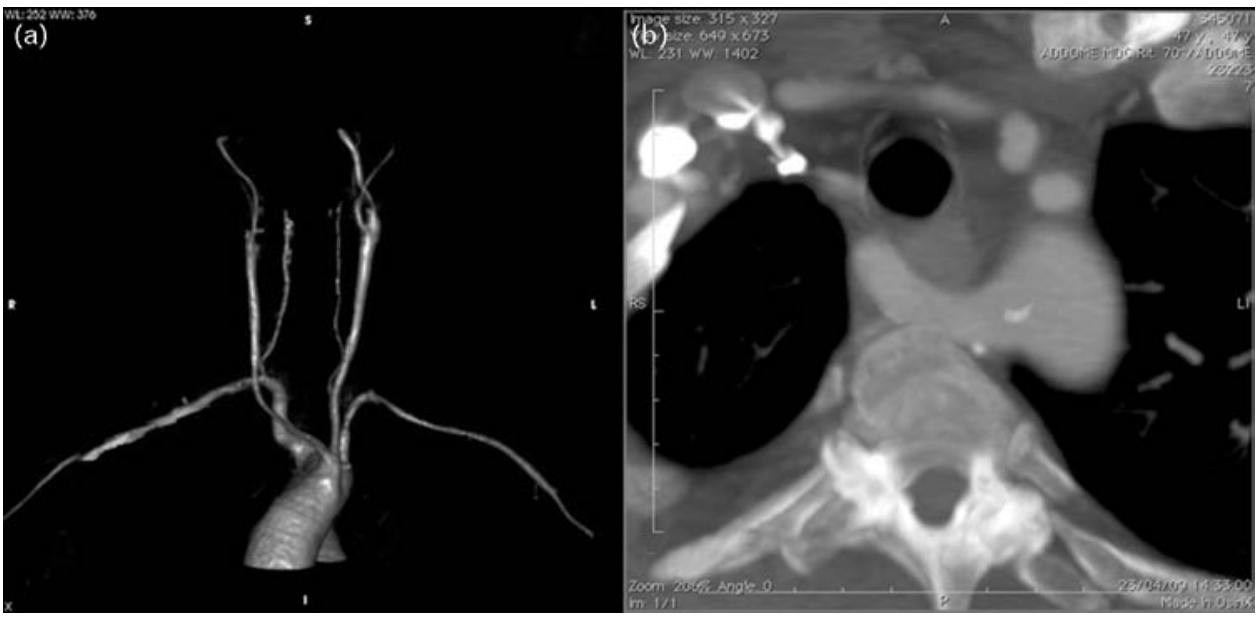

(a) A volume-rendered three-dimensional computed tomography angiography reconstruction of the aortic arch showing origination of common carotid trunk, left subclavian artery and aberrant right subclavian artery. Left internal carotid stenosis and right internal carotid occlusion are also noted. (b) Contrast-enhanced axial CT image demonstrating common carotid trunk anterior to the trachea and aberrant right subclavian artery posterior to the esophagus.

The combination of these two aortic arch anomalies and the concomitant treatment of atherosclerotic carotid stenosis have not been described previously. Chahwan et al. [4] found a similar aortic arch variant in a 75-year-old man with left-sided paresthesias. The patient underwent a diagnostic arteriogram that revealed a common trunk for both carotid arteries, an aberrant RSCA originating directly from the middle of the aortic arch and a moderate stenosis of the right ICA that was managed with antiplatelet therapy. In addition to the aortic arch anomaly, our patient presented a severe symptomatic carotid stenosis associated with contralateral carotid occlusion and ipsilateral vertebral stenosis. This represents an extremely challenging therapeutic dilemma. In the
North American Symptomatic Carotid Endarterectomy Trial (NASCET), indeed, an occluded contralateral carotid artery significantly increased the risk of stroke [5]. On the other hand, anomalies of the aortic arch and its branches are correlated with an increased risk of neurological complications during carotid artery stenting (CAS) [6]. We decided to treat the patient endovascularly for two main reasons: first, the brain was exposed to relative short periods of ischemia (up to $5 \mathrm{~s}$ ); and second, the stenotic lesion was long and high so that a shunt placement during CEA was considered to be technically difficult.

Finally, preoperative CTA with three-dimensional reconstruction of the aortic arch and carotid arteries appears to

Fig. 2
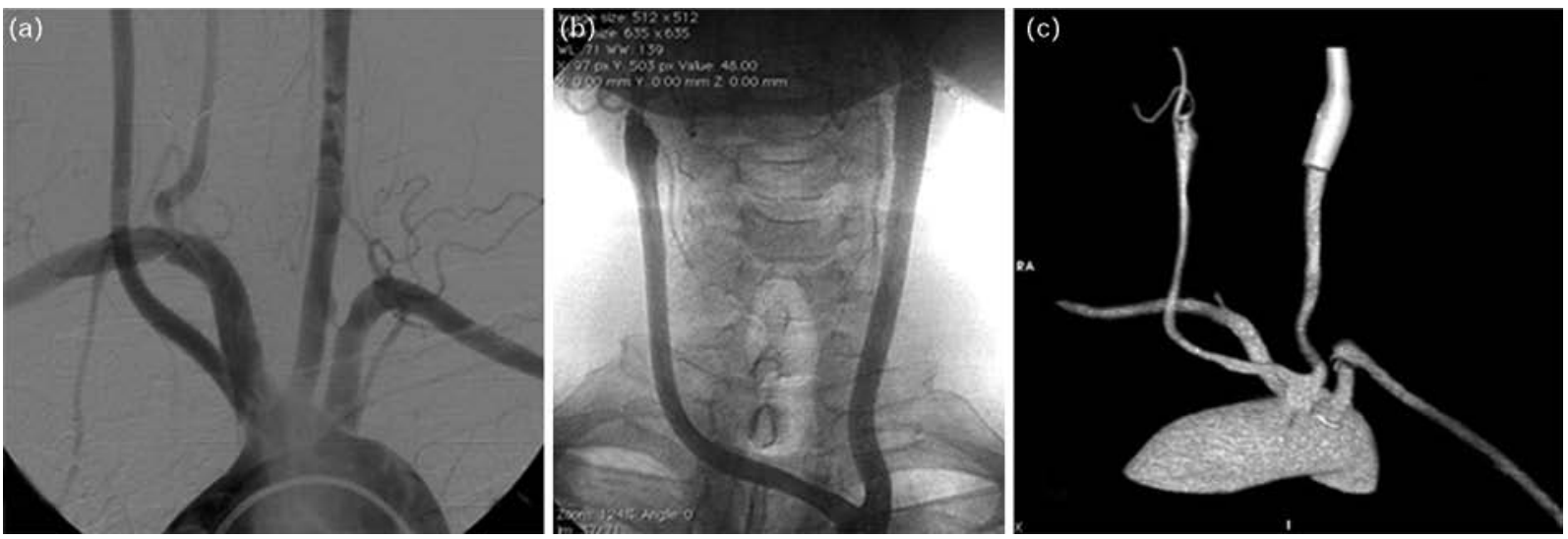

(a) Aortic arch angiogram showing vessel anomalies and left vertebral ostial stenosis. (b) Completion angiogram of the common carotid trunk after deployment of a Carotid Wallstent at level of left carotid bifurcation. (c) Three-month follow-up computed tomography angiography. 
Fig. 3
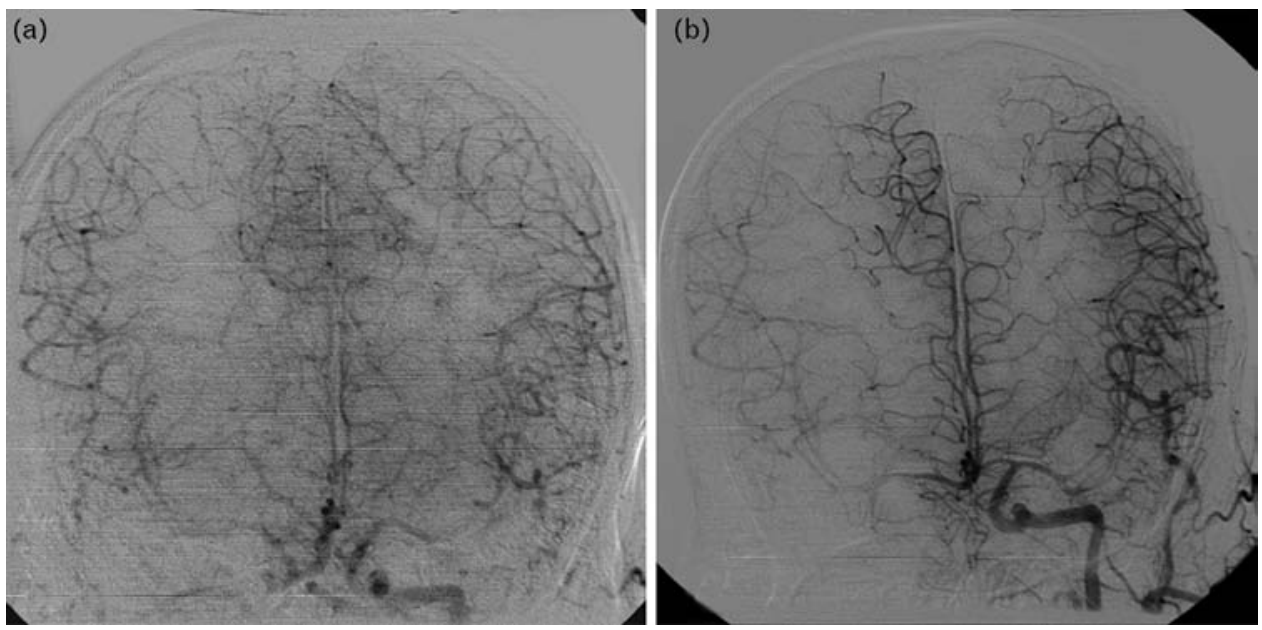

(a) Preprocedural intracranial angiogram through pigtail diagnostic catheter positioned in the aortic arch. (b) Poststenting angiogram of the intracranial circulation through the sheath positioned in left common carotid artery.

be a useful and noninvasive tool for the assessment prior to carotid stenting [7], mostly in determination of vascular anomalies, as the vascular anatomy can be shown with high resolution facilitating the intraoperative catheterization.

\section{References}

1 Jan SL, Hwang B, Fu YC, Chi CS. A rare vascular ring caused by an aberrant right subclavian artery concomitant with the common carotid trunk: a report of two cases. Int $J$ Cardiovasc Imaging 2006; 22:253-256.

2 Natsis KI, Tsitouridis IA, Didagelos MV, Filippidis AA, Vlasis KG, Tsikaras PD. Anatomical variations in the branches of the human aortic arch in 633 angiographies: clinical significance and literature review. Surg Radiol Anat 2009; 31:319-323.
3 Ozateş M, Nazaroglu $\mathrm{H}$, Uyar A. MR angiography in diagnosis of aberrant right subclavian artery associated with common carotid trunk. Eur Radiol 2000; 10:1503.

4 Chahwan S, Miller MT, Kim KA, Mantell M, Kirksey L. Aberrant right subclavian artery associated with a common origin of carotid arteries. Ann Vasc Surg 2006; 20:809-812.

5 Gasecki AP, Eliasziw M, Ferguson GG, Hachinski V, Barnett HJ. Long-term prognosis and effect of endarterectomy in patients with symptomatic severe carotid stenosis and contralateral carotid stenosis or occlusion: results from NASCET. North American Symptomatic Carotid Endarterectomy Trial (NASCET) Group. J Neurosurg 1995; 83:778-782.

6 Faggioli GL, Ferri M, Freyrie A, Gargiulo M, Fratesi F, Rossi C, et al. Aortic arch anomalies are associated with increased risk of neurological events in carotid stent procedures. Eur J Vasc Endovasc Surg 2007; 33:436-441.

7 Wyers MC, Powell RJ, Fillinger MF, Nolan BW, Cronenwett JL. The value of 3D-CT angiographic assessment prior to carotid stenting. J Vasc Surg $2009 ; 49: 614-622$. 